\title{
Impacto econômico da condenação de carcaças por Tuberculose no Baixo Amazonas, Pará, Brasil
}

\section{Economic impact of condemnation of tuberculosis carcasses in the Lower Amazon, Pará, Brazil}

\author{
Giovanne Gabriel Freitas Dourado ${ }^{1}$, Breno Parkerson Martins Alvarenga ${ }^{1}$, Brena Peleja \\ Vinholte $^{1}$, Welligton Conceição da Silva ${ }^{2}$, Albiane Sousa de Oliveira ${ }^{3}$, Hipócrates de Menezes \\ Chalkidis ${ }^{1}$, Éder Bruno Rebelo da Silva ${ }^{2}$, Raimundo Nonato Colares Camargo Júnior ${ }^{4 *}$
}

\begin{abstract}
RESUMO
Objetivou-se estimar o prejuízo econômico oriundo de carcaças de bovinos e bubalinos condenados por tuberculose, durante o ano de 2018, na microrregião de Santarém, na mesorregião do Baixo Amazonas, no estado do Pará, Amazônia Oriental. Os dados foram fornecidos pela Secretaria Municipal de Agricultura e Pesca, no setor de inspeção municipal, Santarém, Pará. Estes continham informações de três abatedouros frigoríficos sobre Serviço de Inspeção Municipal, sobre a espécie bovina e bubalina, especificamente: sexo e o total de animais abatidos e condenados. Foram inspecionados 23.963 bovinos e bubalinos no ano de 2018, provenientes de municípios da mesorregião do Oeste do Pará. Calculou-se também a prevalência de condenação e os prejuízos econômicos. Ao todo foram condenadas por tuberculose 74 carcaças de bovinos e bubalinos. Desse quantitativo, 43 eram fêmeas e 31 machos. A prevalência foi de $0,006 \%$. As perdas econômicas apresentadas em ordem decrescente devido à condenação de carcaça no rebanho, para fêmeas e para machos, foram equivalentes a $\mathrm{R} \$ 293.040,00 ; \mathrm{R} \$ 170.280,50$ e $\mathrm{R} \$ 122.760,00$, respectivamente. Conclui-se que a prevalência foi baixíssima para a tuberculose e o prejuízo econômico decorrente desta enfermidade foi significativo, necessitando-se investigar o prejuízo econômico que as diversas enfermidades causam no Baixo Amazonas para mitigar as perdas futuras decorrentes desta e de outras enfermidades.
\end{abstract}

Palavras-chave: Prevalência; Prejuízo econômico; Condenação de carcaças; Bovinos; Bubalinos.

\footnotetext{
ABSTRACT

This study aimed to estimate the economic loss from carcasses of bovine and buffaloes condemned by tuberculosis, in 2018, in the microregion of Santarém, within the mesoregion of Baixo Amazonas, in the state of Pará, Eastern Amazon. Data were provided by the Municipal Department of Agriculture and Fisheries, in the municipal inspection sector, Santarém, Pará. These contained information from three slaughterhouses on the Municipal Inspection Service, about the bovine and buffalo species, specifically: sex and the total number of animals slaughtered and condemned. 23,963 cattle and buffaloes were inspected

${ }^{1}$ Centro Universitário da Amazônia (UNAMA). E-mail: giiovanne.dourado@ gmail.com; brenoparkeson@gmail.com; brenavinholte@ hotmail.com; chalkidis@ hotmail.com ${ }^{2}$ Universidade Federal Rural da Amazônia (UFRA). E-mail: welligton.medvet@gmail.com; eder.b.rebelo@gmail.com

${ }^{3}$ Universidade Federal do Mato Grasso do Sul. E-mail: albianeoliveiramedvet@hotmail.com

${ }^{4}$ Instituto Federal de Educação, Ciência e Tecnologia do Pará (IFPA). *E-mail: camargojunior@gmail.com
} 
in 2018, from municipalities in the western region of Pará. The prevalence of condemnation and economic losses was also calculated. Altogether, 74 carcasses of cattle and buffaloes were convicted of tuberculosis. In which 43 were elaborated and 31 males. The prevalence was $0.006 \%$. Economical losses presented in descending order due to carcass condemnation in the herd, to estimate and for males, were equivalent to $\mathrm{R} \$ 293,040.00$; $\mathrm{R} \$ 170,280.50$ and $\mathrm{R} \$ 122,760.00$, respectively. It is concluded that the prevalence of tuberculosis was very low and the economic loss resulting from this disease was significant, making it necessary to investigate the economic loss that the various diseases cause in the Lower Amazon Region to mitigate future losses resulting from this and other diseases.

Keywords: Prevalence; Economic loss; Conviction of carcasses; Cattle; Buffaloes.

\section{INTRODUÇÃO}

A carne vermelha faz parte da alimentação dos brasileiros, sendo um alimento bastante procurado pela população (Silva et al., 2020; Abreu et al., 2021; Silva et al., 2021). A produção de carne no Brasil é o setor que vem apresentando um grande crescimento nos últimos anos. Somente em 2019, o Brasil exportou cerca de 1.866.476 toneladas de carne bovina, ao passo que, em 2020, houve um aumento no número de toneladas para 2.012.972 (ABIEC, 2020).

Assim, dado a importância que este alimento representa, é imprescindível que haja um rígido controle sanitário da carne em toda sua cadeia produtiva pois a tuberculose representa risco à saúde pública e segundo Sousa et al. (2021), o Brasil está entre os 22 países prioritários para gerência e controle da Tuberculose em humanos.

Dentre as principais enfermidades que afetam o rebanho de bovinos e bubalinos destaca-se a tuberculose, que causa prejuízos econômicos consideráveis (Melo et al., 2017). Segundo Sa'idu et al. (2015), a região norte do Brasil, em especial o estado do Pará, possui o maior rebanho bubalino e o quinto maior de bovinos, no ranking brasileiro.

A transmissão dessa doença entre bovídeos ocorre especialmente pelo trato respiratório, através da inalação de aerossóis oriundas de animais, além de água, pastagens e alimentos contaminados (Phepa et al., 2016). Outro fator importante para a propagação da enfermidade está relacionado à compra de animais não testados, pois quando se faz aquisição de bovinos e bubalinos infectados estes podem funcionar como fonte de infecção para o rebanho, causando a disseminação da enfermidade dentro das propriedades (Conlan et al. 2012, Mosavari et al. 2016). 
A tuberculose em bovinos e bubalinos tem como principal agente causador 0 Mycobacterium Bovis (Kozakevich e Silva, 2016). É caracterizada pelo surgimento progressivo de lesões nodulares que se localizam em qualquer órgão ou tecido do corpo, o que pode ocasionar variados sinais clínicos e diferentes manifestações da doença (Alberton, 2021).

Geralmente as manifestações da doença se apresentam tardiamente sendo, na maioria dos casos, a necropsia e a avaliação post mortem de carcaças, os principais métodos para a confirmação do diagnóstico (Ruggiero et al. 2007).

Diante desse cenário, observa-se a importância da tuberculose para a produção animal. Assim, este estudo teve como objetivo estimar o prejuízo econômico oriundo de carcaças de bovinos e bubalinos condenados por tuberculose, durante o ano de 2018, na microrregião de Santarém, na mesorregião do Baixo Amazonas, no estado do Pará, Amazônia Oriental.

\section{MATERIAL E MÉTODOS}

Os dados foram coletados na Secretaria Municipal de Agricultura e Pesca (SEMAP), no setor de inspeção municipal, do município de Santarém, Pará, Brasil. Foram utilizadas as informações de três abatedouros frigoríficos (A, B e C) que funcionam sob a fiscalização do Serviço de Inspeção Municipal (SIM).

Os estabelecimentos supramencionados abatem somente bovinos e bubalinos. Neste estudo as duas espécies foram consideradas como um só rebanho, pois para fins de análise, foram considerados especificamente sexo e o total de animais abatidos - para ambas as espécies - cujas carcaças foram condenadas por tuberculose. O sexo foi identificado por meio do Guia de Trânsito Animal (GTA).

Foram inspecionadas 23.963 carcaças de bovinos e bubalinos estudadas no período de janeiro a dezembro de 2018. Os animais eram provenientes de municípios da microrregião de Santarém, que engloba oito munícipios distintos e que está contida na mesorregião do Baixo Amazonas, no estado do Pará - Amazônia Oriental.

As condenações foram realizadas pelo SIM com base na inspeção das lesões macroscópicas sugestivas de tuberculose, presentes no interior das carcaças ou em diferentes órgãos avaliados (pulmão, linfonodos, fígado e baço), conforme preceitua o Art.171 do RIISPOA (Brasil, 2017). 
Para o cálculo de prevalência foram considerados o total de condenações por tuberculose para os três municípios em comparação ao total de animais abatidos no período observado.

$$
P=\frac{\text { Número de indivíduos condenados por tuberculose }}{\text { Total de indivíduos estudados }}
$$

Para estimar o prejuízo econômico causado pela condenação das carcaças do rebanho, em valores atualizados, o preço atribuído ao quilograma da carne foi $\mathrm{R} \$ 18,00$, que equivale ao valor médio - calculado considerando-se as duas espécies e os dois sexos; pago pelos frigoríficos durante o mês de novembro de 2021.

Neste estudo, para fins de cálculo de estimativa do prejuízo econômico, foram avaliadas apenas as condenações totais de carcaças. Para tanto atribuiu-se o peso da carcaça $(220 \mathrm{~kg})$, que equivale ao peso médio calculado, melhor dizendo, $220 \pm 35 \mathrm{~kg}$; obtido a partir do cálculo do rendimento médio de carne por carcaça para as duas espécies e para os dois sexos, para todo o rebanho estudado.

Deste modo, o prejuízo econômico foi estimado da seguinte maneira:

$$
P E_{\text {ano }}=Q C C T_{\text {ano }} \times R M C C \times P M_{K g}
$$

Em que:

$\mathrm{PE}_{\mathrm{ano}}=$ Prejuízo econômico durante o ano de 2018;

QCCT $_{\text {ano }}=$ Quantidade de carcaças condenadas totalmente no ano de 2018; $\mathrm{RMCC}=$ Rendimento médio de carne por carcaça; e,

$\mathrm{PM}_{\mathrm{Kg}}=$ Preço médio pago pelos frigoríficos durante o mês de novembro de 2021 .

Após a coleta, os dados foram tabulados e posteriormente organizados em planilhas do programa Microsoft Excel ${ }^{\circledR}$ 2017, sendo expostos em dados percentuais.

\section{RESULTADOS}

Foram observadas 23.963 carcaças, destas 148 foram condenadas. Dentre as quais $50 \%$ foram condenadas por tuberculose, das quais 43 eram fêmeas. 
Nesta investigação, a prevalência de condenação de carcaças por tuberculose dos animais provenientes do Baixo Amazonas, no período observado, foi baixíssima, equivalendo a aproximadamente $0,006 \%$. Resultado muito inferior ao observado por Kantor e Ritacco (2006) que referenciam $0,37 \%$ de prevalência de tuberculose nos animais estudados na região Centro-Oeste.

Foi possível observar uma diferença na proporção de tuberculose entre os sexos, na qual o maior índice de condenações foi registrado para as fêmeas que responderam por 57,1\%. Estes resultados concordam com aqueles apresentados por Pereira et al. (2017), realizados na mesma região, o qual reportou maior ocorrência de tuberculose em fêmeas bovinas $(87,6 \%)$ e bubalinas $(71,4 \%)$. Segundo Todeschini et al. (2018) as fêmeas apresentam maiores chances de contrair a enfermidade; tendo em conta que a maior parte dos animais abatidos eram fêmeas e que estas tendem a permanecer mais tempo no rebanho, a soma destes fatores supostamente facilitaria a infecção e, por conseguinte, o resultado ora apresentado.

Apesar disso, Torres e Dreher (2015) sinalizam que o abate excessivo de vacas pode acarretar diminuição no número de neonatos e aumentar o índice de animais jovens no plantel, fato que se revela positivo para Santos e Lopes (2014); segundo estes, a renovação de matrizes dentro do rebanho é de fundamental importância, pois os animais que serão substitutos apresentariam elevado potencial produtivo. Segundo Radostitis et al. (2002) provavelmente o menor número de infecção nos machos pode estar relacionado ao fato destes ficarem isolados em boa parte do ano.

As perdas econômicas - apresentadas em ordem decrescente - decorrentes da condenação de carcaça no rebanho, para fêmeas e para machos, foram equivalentes a R \$ 293.040,00; R\$ 170.280,50 e R\$122.760,00, respectivamente.

No Baixo Amazonas não há estudos estimando perdas econômicas por carcaça, ainda que por outras causas, que não a tuberculose. Deste modo, esta investigação revelou-se pioneira nesta metodologia e por isso não há dados sobre este aspecto que possam ser confrontados com os resultados obtidos.

O que mais se aproxima deste escopo estudou perdas econômicas por vísceras, realizado por Pereira et al. (2020), os quais encontraram prejuízos na ordem de R\$ 241.701,99; sinalizando perdas econômicas consideráveis, com valores aproximados aos desta pesquisa. 
De um modo geral, as condenações por enfermidades causam prejuízos severos para a produção animal e a cadeia produtiva da carne. Isto pode ser observado em diversos estudos, sobre os mais diversos temas, conforme aduz Souza, Campos e Procópio (2018).

Esta equipe estudou a perda econômica por cisticercose para os estados que compõem a região Centro-Oeste, no período de 2013 a 2017 e referenciou - em ordem crescente de valores, $\mathrm{R} \$ 1.656 .769,35 ; \mathrm{R} \$ 31.563,87$ e $\mathrm{R} \$ 34.212 .237,94$; para os estados de Mato Grosso, Goiás e Mato Grosso do Sul, respectivamente. Note-se que os valores apresentados se referem aos respectivos estados durante cinco anos.

Ainda no centro-Oeste, precisamente no estado de Goiás, Aquino (2017), estima perdas para o período de 2007 a 2014, na ordem de $\mathrm{R} \$ 64.809 .817,50$; devido à presença da cisticercose no rebanho e cerca de $\mathrm{R} \$ 15.072,75$ reais; devido à presença de Fasciolose em bovinos.

Bidone et al. (2021), referencia para o período de 2015 a 2018, o valor perdido em virtude da condenação de abate em estabelecimentos sob inspeção estadual no Rio Grande do Sul, uma média anual de US\$ 8.949.000,00; US\$ 3.539.000,00; US\$ 12.847.000,00 e US\$ 4.798.000,00; para as seguintes enfermidades: Cisticercose, Hidatidose, Fasciolose e Esofagostomose, respectivamente.

A exemplo da equipe anterior e ainda diferentemente deste estudo, a pesquisadora também avaliou as perdas estaduais, o que pode justificar a diferença exorbitante de valores desta para aquela pesquisa.

Para o mesmo estado, Dick et al. (2019), aponta prejuízo estimado em 2,35 milhões de dólares por ano, no período de 2007 a 2015, para diversas causas. Dentre elas, vale destacar que a tuberculose foi responsável por $17,43 \%$ das perdas verificadas.

\section{CONCLUSÃO}

Esta investigação apontou uma prevalência baixíssima para a tuberculose. Não obstante, o prejuízo econômico decorrente desta enfermidade foi significativo. Ainda que esta pesquisa seja pioneira, está clara a necessidade de se investigar o prejuízo econômico que as diversas enfermidades causam no Baixo Amazonas para mitigar as perdas futuras decorrentes desta e de outras enfermidades. 


\section{REFERÊNCIAS}

ABIEC. Associação Brasileira das Indústrias Exportadoras de Carne. 2020. Disponível em: \&lt; http://abiec.com.br/ \&gt;. Acesso em 27 de Março. 2021.

Abreu ML, Dantas GS, Silva WC, Barbosa AVC, Silva JAR. Percepção dos consumidores de proteína animal sobre o bem-estar dos animais de produção no município de Parauapebas, Pará, Brasil. Veterinária e Zootecnia, v. 28, p. 1-12, 2021. DOI: https://doi.org/10.35172/rvz.2021.v28.484

ALBERTON, L. F. S. Tuberculose bovina - métodos de diagnóstico, tratamento, controle e prevenção: Revisão de Literatura. 2021. 34 f. Trabalho de Conclusão de Curso (graduação) - Universidade Federal de Santa Catarina, 2021.

AQUINO, F. M. Prevalência e distribuição espacial da Cisticercose e Fasciolose bovina no estado de Goiás. 2017. 113 f. Dissertação (Mestre em Ciência Animal) Universidade Federal de Goiás.

BIDONE, N. D. B., GONÇALVES, A. P., BREMM, C., GIROTTO, A. F., GIROTTOSOARES, A., SANTOS, P., ... \& SOARES, J. F. Condenação ao abate em bovinos devido a lesões parasitárias e seu impacto econômico em estabelecimentos do Sistema Federal de Inspeção no Brasil e no Sistema Estadual de Inspeção no Rio Grande do Sul. Revista Brasileira de Parasitologia Veterinária, v. 30, n. 1, 2021.

CONLAN, A. J.; MCKINLEY, T. J.; KAROLEMEAS, K.; POLLOCK, E. B.; GOODCHILD, A. V.; MITCHELL, A. P.; BIRCH, C. P.; CLIFTON-HADLEY, R. S.; WOOD, J.L. Estimating the hidden burden of bovine tuberculosis in Great Britain. PLOS Computational Biology, v. 8, n. 1, p. 1-14, 2012.

DICK, M.; SILVA, M. A.; SILVA, R. R. F.; SOUZA, J. P.; FERREIRA, O. G. L.; MAIA, M. S. Impactos econômicos da condenação de carcaças bovinas no Sul do Brasil. Archivos Latinoamericanos de Producción Animal, v. 27, p. 1-4, 2019.

KANTOR, I. N.; RITACCO, V. An update on bovine tuberculosis programmes in Latin American and Caribbean countries. Veterinary Microbiology, v. 112, p. 111-118, 2006. 
KOZAKEVICH, G. V.; SILVA, R. M. Tuberculose: revisão de literatura. Arquivos Catarinenses de Medicina, v. 44, n. 4, p. 34-47, 2016.

MOSAVARI, N.; GERAVAND, M. M.; TADAYONA, K.; KESHAVARZ, R. Mycobacterial coinfection and persisting bovine tuberculosis - Has the time arrived for a policy review? International Journal of Mycobacteriology, v. 5, n. 1, p. 82-83, 2016.

PEREIRA, M. F.; CIRNE, L. G. A.; NEVES, K. A. L.; CLAUDIANO, G. S.; COSTA, A.S.; CASTRO, E. K. F.; MORINI, A. C.; CARVAlHO, G. G. P. Condenações de bovídeos abatidos sob inspeção municipal em Santarém - PA. Agroecossistemas, v. 9, n. 2, p. 78-90, 2017.

PEREIRA, P. A. R., SOARES, M. DE C., BATISTA, H. R., SILVA, W. C. DA, \& CAMARGO JUNIOR, R. N. C. Study of bovine heart, liver and lung condemnations occurred in the municipality of Santarém, Pará. Revista de Ciência Veterinária e Saúde Pública, v. 7, n. 2, p. 077-083, 2020. https://doi.org/10.4025/rcvsp.v7i2.54161

RADOSTITS, O. M.; GAY, C. C.; BLOOD, D. C.; HINCHCLIFF, K. W. Clínica Veterinária: um tratado de doenças dos bovinos, ovinos, suínos, caprinos e equinos. 9 ed. Rio de Janeiro: Guanabara Koogan, 2002, p. 1772.

RUGGIERO, A. P.; IKUNO, A.A.; FERREIRA, V.C.A.; ROXO, E. Tuberculose bovina: alternativas para o diagnóstico. Arquivos do Instituto Biológico, v. 74, n. 1, p. 55-65, 2007.

PHEPA, P. B.; CHIROVE, F.; GOVINDER, K. S. Modelling the role of multitransmission routes in the epidemiology of bovine tuberculosis in cattle and buffalo populations. Mathematical Biosciences, v. 277, p. 47-58, 2016.

SA'IDU, A.S.; OKOLOCHA, E.C.; DZIKWI, A.A.; GAMAWA, A.A.; IBRAHIM, S.; KWAGA, J.K.P.; USMAN, A. \& MAIGARI, S.A. Public health implications and risk factors assessment of Mycobacterium bovis infections among abattoir personnel in Bauchi state, Nigeria. Journal of Veterinary Medicine, v. 2015, n. 1, p. 1-5, 2015.

SANTOS, G.; LOPES, M. A. Indicadores econômicos de sistemas de produção de leite em confinamento total com alto volume de produção diária. Ciência Animal Brasileira, v. 15, p. 239-248, 2014. 
SILVA, C. A. S.; JOSET, W. C. L.; LOURENÇO JÚNIOR, J. B.; BARBOSA, A. V. C.; SILVA, W. C.; SILVA, J. A. R. Animal protein consumer's perception on the welfare of production animals in Belém, Pará State, Brazil. Acta Scientiarum. Animal Sciences, v. 43, n. 1, p. e53784, 2021. https://doi.org/10.4025/actascianimsci.v43i1.53784

SILVA, W. C.; SILVA, J. A. R.; SILVA, E. B. R. Percepção do consumidor de proteína animal sobre o bem-estar dos animais de produção em Santarém, Pará, Brasil. Revista CES Medicina Veterinaria y Zootecnia, v. 15, n. 2, p. 64-74, 2020. DOI: http://dx.doi.org/10.21615/cesmvz.15.2.5

SOUSA, M. N. A. de, SILVA, A. M. M. da ., ALVES, P. R. F. ., FAUSTO, P. F. ., DIAS, T. R. ., SUAREZ, L. de A. B., MACEDO, M. B., \& BEZERRA, A. L. D. (2021). Fatores de risco e tuberculose: alerta para os profissionais de saúde. Conjecturas, 21(4), 785-796. https://doi.org/10.53660/CONJ-289-315

SOUZA, W. F.; CAMPOS, G. A.; PROCÓPIO, D. P. Aspectos econômicos e sanitários sobre a condenação total de carcaças bovinas por Cisticercose no estado de Mato Grosso do Sul. ENCICLOPÉDIA BIOSFERA, Centro Científico Conhecer, v.15 n. 28, p. 1-7, 2018. DOI: 10.18677/EnciBio_2018B11

TODESCHINI, B; COSTA, E. F.; NETO, W. S.; SANTOS, D. V.; GROFF, A. C. M.; BORBA, MAURO, R.; CORBELLINI, L. G. Ocorrência de brucelose e tuberculose bovinas no Rio Grande do Sul com base em dados secundários. Pesquisa Veterinária Brasileira, v. 38, n. 1, p. 15 - 22, 2018.

TORRES, R. N. S.; DREHER, A. Uso de fêmeas (novilhas e vacas de descarte) para a produção de carne aspectos produtivos e qualidade. Revista Eletrônica Nutritime, v. 12, n. 1, p. 4082 - 4089, 2015.

MELO, E. H. Estudo retrospectivo da tuberculose bovina diagnosticada na Clínica de Bovinos de Garanhuns, PE, no período de 2009 a 2016. Revista Acadêmica Ciência Animal, v. 15, p. 39-40, 2017.

Recebido em: 11/10/2021

Aprovado em: 20/11/2021

Publicado em: 25/11/2021 